\title{
Breve reseña histórica de la primera publicación del síndrome de insuficiencia respiratoria aguda
}

\author{
Brief historical review of the first publication of the acute respiratory insufficiency \\ syndrome
}

Raúl Carrillo-Esper ${ }^{1 *}$ y Dulce M. Carrillo-Córdova²

${ }^{1}$ Academia Nacional de Medicina, Academia Mexicana de Cirugía, Instituto Nacional de Rehabilitación Dr. Luis Guillermo Ibarra Ibarra; 2 Instituto Nacional de Ciencias Médicas y Nutrición Dr. Salvador Zubirán, Ciudad de México, México

\section{Resumen}

El síndrome de insuficiencia respiratoria aguda fue descrito por primera vez en la revista The Lancet en el año 1967 por Ashbaugh, Petty, Levine y Bigellow. Su estudio se fundamenta en la descripción de una serie de 12 enfermos que presentaban insuficiencia respiratoria aguda, cianosis refractaria a tratamiento con oxígeno, disminución de la distensibilidad pulmonar e infiltrados difusos en la radiografía de tórax. La mortalidad fue del 58\%, con más oportunidad de sobrevida en aquellos enfermos manejados con ventilación mecánica y presión positiva al final de la espiración. El estudio histopatológico mostró pulmones de mayor peso, atelectasias, edema intersticial y alveolar, y membranas hialinas. A 50 años de su publicación, se han logrado importantes avances en el conocimiento y el tratamiento de esta enfermedad, lo que se ha reflejado en una disminución de la mortalidad. El objetivo de este trabajo es describir la publicación original y reconocer su importancia y la genialidad de los autores, para de esta manera celebrar el cincuentenario de este artículo clave y fundamental en la medicina intensiva y hacer un justo homenaje a los que participaron en su publicación.

PALABRAS CLAVE: Síndrome de insuficiencia respiratoria aguda. Presión positiva al final de la espiración. Ventilación mecánica.

\begin{abstract}
Acute respiratory distress syndrome was first described by Ashbaugh, Petty, Levine y Bigellow in 1967 writing in The Lancet. Their study was based on a case series of 12 patients with acute respiratory distress, cyanosis refractory to oxygen therapy, decreased lung compliance and diffuse infiltrates evident on the chest radiograph. Mortality was $58 \%$ with greater survival in five patients managed with mechanical ventilation and positive end expiratory pressure. Histopathology revealed heavy lungs, atelectasis, interstitial and alveolar edema, as well as hyaline membranes. 50 years after the publication of this article, advances in knowledge and management of this disease have been considerable, which is reflected in decreased mortality. The aim of this paper is to describe the original publication, recognize its importance and genious of its authors, to celebrate the $50^{\text {th }}$ anniversary of this landmark and fundamental paper in intensive care medicine and honor their authors.
\end{abstract}

KEY WORDS: Acute respiratory distress syndrome. Positive end expiratory pressure. Mechanical ventilation.

\author{
Correspondencia: \\ *Raúl Carrillo-Esper \\ Calzada México-Xochimilco, 289 \\ Col. Arenal Tepepan, Del. Tlalpan \\ C.P. 14389, Ciudad de México, México \\ E-mail: revistacma@ comexane.org
}

Fecha de recepción: 13-07-2018

Fecha de aceptación: 13-10-2018

DOI: 10.24875/CIRU.18000592
Cir Cir. 2019;87:113-122

Contents available at PubMed www.cirugiaycirujanos.com 


\section{A 50 años de la primera publicación del síndrome de insuficiencia respiratoria aguda}

La medicina intensiva es una especialidad apasionante, $y$ todos los que la practicamos hemos sido testigos de cómo en pocos años se han logrado grandes avances que han hecho posible la sobrevida de un gran número de enfermos. Del conocimiento fisiopatológico y el mecanicismo reduccionista en los que se basaba la toma de decisiones, se ha evolucionado a las bases genéticas, moleculares y a los grandes ensayos clínicos controlados, que han señalado en los últimos años el camino a seguir, mostrando en muchos casos que la traslación a la clínica de conceptos básicos no siempre da los resultados que se pensaba.

En los años 1960 la medicina intensiva daba sus primeros pasos, pero este decenio fue fundamental debido a que se sentaron las bases de la especialidad y se profundizó en el estudio de algunas enfermedades que, por sus características, comportamiento y elevada mortalidad, tenían que ser evaluadas dinámicamente y atendidas en salas especiales por un nuevo gremio de especialistas: los intensivistas.

El objetivo de este trabajo es celebrar el cincuentenario de una publicación que revolucionó la medicina intensiva y la ventilación mecánica.

\section{La publicación}

El sábado 12 de agosto de 1967 es una fecha histórica para la medicina intensiva, pues en la revista The Lancet se publicó un trabajo parteaguas que fue titulado por sus autores Acute respiratory distress in adults ${ }^{1}$.

\section{Acute respiratory distress in adults}

Este artículo tiene una extensión de cinco cuartillas, que van de la página 319 a la 323 del volumen 2 de la revista científica The Lancet. Además, el escrito incluye tres tablas, cinco figuras y 22 referencias bibliográficas. Su trascendencia radica en que sentó las bases no solo del conocimiento de una enfermedad de la que se tenían antecedentes pero que no era integrada como un síndrome específico y que en la actualidad conocemos como síndrome de insuficiencia respiratoria aguda (SIRA), al que los autores se refieren en la publicación como "dificultad respiratoria aguda en adultos», sino también del desarrollo de la ventilación mecánica y de la presión positiva al final de la espiración (PPFE). Al momento de su publicación ya se tenían algunos avances en el conocimiento de la lesión pulmonar en el estado de choque, pues ya en la década de 1940 se habían descrito atelectasias y congestión en pacientes que morían de choque hemorrágico, lo que denominó Jenkins en los años 1950 como síndrome de atelectasias congestivas. En 1959, Petersdorf describió las lesiones pulmonares de enfermos que murieron de influenza y las comparó con las de la pandemia que se presentó en 1914-1918, observando que en ambos brotes se presentaban pulmones hemorrágicos, con pérdida de volumen aéreo, y en el estudio histológico se encontraba congestión edematosa, necrosis alveolar y membranas hialinas. A partir de la segunda mitad de los años 1950 se hicieron las consideraciones teóricas de la importancia y la función del surfactante, y del efecto tóxico del oxígeno a elevadas concentraciones. En la Segunda Guerra Mundial y en la Guerra de Vietnam se hizo evidente la lesión pulmonar que se presentaba en soldados heridos en combate que sobrevivían a la lesión inicial y que eran reanimados agresivamente, lo que se denominó «pulmón de choque» 0 "pulmón húmedo", y en la Guerra de Vietnam «pulmón de Danang", pues se presentaron varios casos en heridos de esta cruenta batalla. La descripción clínica era muy característica: hombres jóvenes heridos en combate, que aun a pesar de no haber sufrido heridas torácicas presentaban un cuadro de dificultad respiratoria, hipoxia y cianosis que no respondía con el aporte de oxígeno, y en la radiografía de tórax se observaba edema que no parecía tener un origen cardiogénico, por lo que se acuñó el término «edema pulmonar no cardiogénico». En el terreno de la ventilación mecánica, en la década de 1960 se contaba con ventiladores Bennet, que basaban su funcionamiento en el flujo de gases (fuente de aire comprimido externo) y válvulas ciclados por presión, y no contaban con otros modos ventilatorios ni alarmas; tecnología muy distante a la ventilación mecánica actual basada en microprocesadores y que ofrece múltiples modos de ventilación y complejos sistemas de monitoreo y alarmas ${ }^{1,2}$ (Figs. 1 y 2 ).

Este era el entorno clínico y científico en el cual se desempeñaban cuatro médicos geniales: David G. Aushbaugh, D. Boyd Bigellow, Thomas L. Petty y Bernard E. Levine, de los departamentos de medicina y cirugía del Centro Médico de la Universidad de Colorado, en Denver (EE.UU.). Sus observaciones las correlacionaron con la evidencia científica con que se contaba al momento y describieron una nueva enfermedad de manera magistral, sentando las bases de lo que ahora es de conocimiento generalizado y adelantándose a 


\section{ACUTE RESPIRATORY DISTRESS IN ADULTS \\ David G. Ashbaugh \\ M.D. Ohio State \\ ASSISTANT PROFESSOR OF SURGERY \\ D. BOYD Bigelow \\ M.D. Colorado}

ASSISTANT IN MEDICINE AND AMERICAN THORACIC SOCIETY-NATIONAL TUBERCULOSIS ASSOCIATION FELLOW IN PULMONARY DISEASE

Thomas L. PetTy

M.D. Colorado

ASSISTANT PROFESSOR OF MEDICINE

BERNARD E. LEVINE

M.D. Michigan

AMERICAN THORACIC SOCIETY-NATIONAL TUBERCULOSIS ASSOCIATION FELLOW IN PULMONARY DISEASE*

From the Departments of Surgery and Medicine, University of Colorado Medical Center, Denver, Colorado, U.S.A.

The respiratory-distress syndrome in 12
patients was manifested by acute onset of
tachypnoa, hypoxamia, and loss of compliance after a
variety of stimuli; the syndrome did not respond to usual
and ordinary methods of respiratory therapy. The clinical
and pathological features closely resembled those seen in
infants with respiratory distress and to conditions in
congestive atelectasis and postperfusion lung. The
theoretical relationship of this syndrome to alveolar
surface active agent is postulated. Positive end-expiratory
pressure was most helpful in combating atelectasis and
hypoxæmia. Corticosteroids appeared to have value in the
treatment of patients with fat-embolism and possibly viral
pneumonia.

Figura 1. Portada del artículo que inició una nueva era en el conocimiento, el abordaje y el tratamiento de la lesión pulmonar aguda.

conceptos como volutrauma, biotrauma, sobredistensión alveolar, PPFE, daño alveolar difuso, comportamiento mecánico pulmonar, patrón evolutivo y efectos de la sobrecarga hídrica en la lesión pulmonar aguda, entre otros.

El contenido de este artículo es sorprendente, ya que solo con 12 pacientes incluidos en el trabajo, de una muestra de 272 enfermos adultos, los autores describen las características clínicas, radiográficas, bioquímicas e histopatológicas de una enfermedad a la que denominaron «dificultad respiratoria aguda en adultos». La descripción clínica que desarrollaron sigue siendo vigente en nuestros días:

..."The clinical pattern includes severe dyspnoea, tachypnoea, cyanosis that is refractory to oxygen therapy, loss of lung compliance, and a diffuse alveolar infiltrate seen on Chest $X$ ray."

Los autores hacen notar, como se muestra en la tabla 1 de la publicación, que el cuadro se inició en las primeras 96 horas del disparador inicial, que ninguno de 


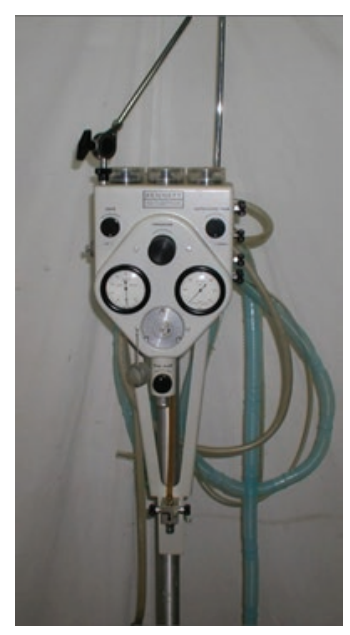

Figura 2. Ventilador Bennet PR-2 utilizado en la década de 1960.

los enfermos cursaban con enfermedad pulmonar crónica y que la etiología era variada: en siete enfermos trauma grave, neumonía viral en cuatro y pancreatitis en uno. Siete de los pacientes presentaron sobrecarga hídrica; en cinco en los que fue calculado, el balance de líquidos fue positivo, oscilando entre 3 y 10.3 litros. Esto refleja que al inicio hubieran sido diagnosticados como insuficiencia cardiaca y manejados con digital. No es de sorprender que la sobrecarga hídrica complicará el cuadro y la evolución de los enfermos, hecho que es bien conocido en la actualidad y que es criterio de exclusión de acuerdo con las definiciones de Berlín y SIESTA (Spanish Initiative for Epidemiology Stratification and Therapies for ARDS) ${ }^{3}$ (Fig. 3).

Los autores describen los infiltrados en parches $y$ bilaterales. Conforme se agravaba la lesión pulmonar, estos se hacían confluentes para posteriormente consolidarse. Puede notarse, en su escrito y en las radiografías que incluyen, la clara definición, basada en la radiografía simple de tórax, de un patrón evolutivo desde congestión, atelectasias y colapso alveolar hacia uno de consolidación con involucro en las bases, que posteriormente se hizo evidente con los estudios de tomografía computada realizados por el Dr. Gattinoni y su grupo cuando se describió el Baby lung:

... "The earliest changes consisted of a patchy, bilateral alveolar infiltrate. These findings were frequently confused with acute heart failure and mild pulmonary oedema. When respiratory distress becomes more severe, the patchy infiltrates become more confluent and before death, $x$-ray appearance was that consolidation."

En relación con los parámetros respiratorios y de ventilación, los autores describen que todos los pacientes cursaban con hipoxemia; a dos los manejaron con oxígeno al aire ambiente, a tres con oxigenoterapia con fracción inspirada de oxígeno del 100\%, y siete fueron intubados y ventilados mecánicamente con presión positiva. La presión parcial de dióxido de carbono en sangre arterial osciló entre 22 y $63 \mathrm{mmHg}$. El gradiente entre la fracción inspirada de oxígeno y la presión arterial de oxígeno en tres enfermos en los que se calculó fue de 536, 320 y $220 \mathrm{mmHg}$. La distensibilidad pulmonar se halló entre 9 y $190 \mathrm{ml} / \mathrm{cmH}_{2} \mathrm{O}$, lo que refleja un rango muy amplio en el volumen corriente, lo que de seguro impactó en amplificar el daño alveolar (algo desconocido en ese tiempo) (Fig. 4).

Se realizó autopsia en siete pacientes, y según describen los autores:

... "At necropsy in seven patients, gross inspection showed heavy and deep reddish-purple lungs ... the appearance resembled liver tissue."

Microscópicamente se observaron hemorragia, congestión, microatelectasias, daño alveolar difuso y membranas hialinas:

... "Microscopic appearance of the lungs was consistent in the 5 patients who died early in the course of the illness. Striking features were hyperaemia, dilated engorged capillaries, and areas of alveolar atelectasis. Interstitial and intra-alveolar haemorrhage and oedema were also common. A striking finding was the presence of hyaline membranes in all but 1 patient. Diffuse interstitial inflammation and fibrosis without notable hyperaemia."

Las intervenciones terapéuticas se clasificaron como de valor dudoso o de valor aparente:

- Se consideraron de valor dudoso el uso de digital, antibióticos, esteroides en caso de lesión pulmonar secundaria a trauma, tolazolina y ventilación con presión positiva intermitente. Describen que el cambio de un modo ventilatorio controlado por presión a uno por volumen mejoraba la ventilación, pero tenía poco impacto en la oxigenación (adelantándose al concepto de volutrauma y sobredistensión alveolar):

... "Patient 9 was ventilated with an experimental volume-cycled respirator incorporating deep breath at regular intervals. Every two minutes the respirator delivered a deep breath that was double the patient's tidal volume. Recovery in this patient may have been partly due to the respirator. The other 11 patients were initially managed on pressure-cycled respirators, which proved inadequate; they would not deliver adequate volumes at the high pressures required. Volume respirators were subsequently used in 7 patients: ventilation improved, but little change was seen in hypoxaemia." 
TABLE I-ACUTE RESPIRATORY DISTRESS

\begin{tabular}{|c|c|c|c|c|c|c|c|}
\hline \multirow{2}{*}{ Case } & \multirow{2}{*}{$\begin{array}{l}\text { Age } \\
\text { (yr.) }\end{array}$} & \multirow{2}{*}{ Sex } & \multirow{2}{*}{ Illness } & \multirow{2}{*}{$\begin{array}{l}\text { Onset of } \\
\text { acute res- } \\
\text { piratory } \\
\text { distress } \\
\text { (hr. after } \\
\text { illness) }\end{array}$} & \multicolumn{3}{|c|}{$\begin{array}{l}\text { Possible contributory } \\
\text { factors }\end{array}$} \\
\hline & & & & & $\begin{array}{l}\text { Hypo- } \\
\text { tension }\end{array}$ & Acidosis & $\begin{array}{c}\text { Fluid } \\
\text { overload }\end{array}$ \\
\hline 1 & 29 & $\mathbf{M}$ & $\begin{array}{l}\text { Multiple trauma; } \\
\text { lung contusion }\end{array}$ & 8 & ++ & ++ & $\begin{array}{c}+++ \\
7500 \mathrm{ml}\end{array}$ \\
\hline 2 & 19 & $\mathbf{F}$ & $\begin{array}{l}\text { Multiple trauma; } \\
\text { lung laceration and } \\
\text { contusion }\end{array}$ & 1 & +++ & ++ & $\begin{array}{l}++++ \\
3000 \mathrm{ml}\end{array}$ \\
\hline 3 & 19 & $\mathbf{F}$ & $\begin{array}{l}\text { Multiple trauma } \\
\text { and fractures; fat- } \\
\text { embolism }\end{array}$ & 72 & + & $\cdots$ & $\cdots$ \\
\hline 4 & 25 & $\mathbf{M}$ & $\begin{array}{l}\text { Shotgun wound to } \\
\text { abdomen }\end{array}$ & 96 & +++ & + & $\begin{array}{l}+++ \\
9000 \mathrm{ml}\end{array}$ \\
\hline 5 & 11 & $\mathbf{M}$ & $\begin{array}{l}\text { Blunt chest injury; } \\
\text { lung contusion }\end{array}$ & 1 & $\cdots$ & ++ & $\cdots$ \\
\hline 6 & 43 & $\mathbf{F}$ & Acute pancreatitis & 48 & +++ & +++ & $\underset{5000 \mathrm{ml}}{++}$ \\
\hline 7 & 23 & $\mathbf{F}$ & ? viral pneumonia & 48 & . & $\cdots$ & $\cdots$ \\
\hline 8 & 39 & $\mathbf{F}$ & $\begin{array}{l}\text { Drug ingestion; } \\
\text { ? viral pneumonia }\end{array}$ & 24 & $\cdots$ & $\cdots$ & ++ \\
\hline 9 & 19 & $\mathbf{F}$ & $\begin{array}{l}\text { Guillain-Barre; } \\
\text { ? viral pneumonitis }\end{array}$ & 96 & $\cdots$ & . & ++ \\
\hline 10 & 18 & $\mathbf{M}$ & $\begin{array}{l}\text { Multiple trauma; } \\
\text { crushed chest; } \\
\text { severe concussion }\end{array}$ & 1 & $\cdots$ & $\cdots$ & $\cdots$ \\
\hline 11 & 48 & $\mathbf{F}$ & $\begin{array}{l}\text { Drug ingestion; } \\
\text { ? aspiration; ? viral } \\
\text { pneumonia }\end{array}$ & 48 & $\cdots$ & $\cdots$ & $\begin{array}{c}+++ \\
10328 \mathrm{ml}\end{array}$ \\
\hline 12 & 34 & $\mathbf{M}$ & $\begin{array}{l}\text { Gunshot wound left } \\
\text { chest }\end{array}$ & 96 & $\cdots$ & $\cdots$ & .. \\
\hline
\end{tabular}

Figura 3. Tabla del artículo original en la que se observan los datos demográficos de la muestra y los balances hídricos. Nótese que en cinco enfermos el balance hídrico es francamente positivo.

... "The value of corticosteroids probably lies in their anti-inflammatory antioedema effect. In our series, corticosteroids were most useful in the management of 1 patient with fat-embolism and in 1 patient with Guillain-Barre paralysis."... "Corticosteroids are of questionable value in the treatment of patients who develop this syndrome after trauma."

- La maniobra que se consideró de valor aparente fue la PPFE. Se tituló entre 5 y $10 \mathrm{cmH}_{2} \mathrm{O}$. De los siete enfermos que no recibieron PPFE, solo dos sobrevivieron. De los cinco que recibieron PPFE, tres sobrevivieron y se recuperaron rápidamente y dos fallecieron, uno de hemorragia y el otro de sepsis (Fig. 5): ... "Continuous positive-pressure respiration was used in the management of 5 patients. End-expiratory pressures were maintained at 5-10 $\mathrm{cm}$ water. All 5 patients demonstrated a rise in $\mathrm{PaO} 2$ or oxygen saturation with the use of end-expiratory pressure." 


\begin{tabular}{|c|c|c|c|c|c|c|c|c|}
\hline Case & Respiratory support & $\begin{array}{c}\text { Frequency } \\
\left(\text { min. }^{-1}\right)\end{array}$ & $\dot{\mathrm{v}}_{\mathrm{E}}(\mathrm{l} . / \mathrm{min})$. & $\mathrm{S}_{2} \mathrm{O}_{3}$ & $\begin{array}{c}\mathrm{P}_{1} \mathrm{O}_{1}-\mathrm{P}_{2} \mathrm{O}_{\mathbf{1}} \\
\text { gradient } \\
\left.\text { (mm. } \mathrm{H}_{\mathbf{g}}\right)\end{array}$ & $\underset{(\mathrm{mm} . \mathrm{Hg})}{\mathrm{P}_{2} \mathrm{CO}_{1}}$ & $\mathrm{pH}$ & $\begin{array}{l}\text { Compliance } \\
\text { (.. } / \mathrm{cm} \text {. } \\
\text { watet) }\end{array}$ \\
\hline $\begin{array}{l}1 \\
2 \\
3 \\
4 \\
5 \\
6 \\
7 \\
8 \\
9\end{array}$ & 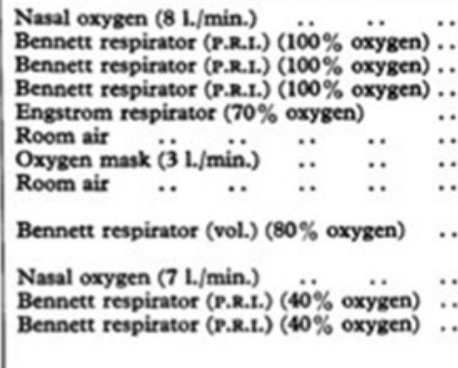 & $\begin{array}{l}40 \\
28 \\
48 \\
48 \\
48 \\
36 \\
64 \\
44 \\
20\end{array}$ & $\begin{array}{c}16-0 \\
16-8 \\
\because . \\
\ddot{80} \\
14-4 \\
\ddot{20}-0 \\
8.0\end{array}$ & $\begin{array}{l}85 \\
72 \\
78 \\
73 \\
85 \\
84 \cdot 4 \\
79 \\
41 . \\
84\end{array}$ & $\begin{array}{c}\because \\
\because \\
\ddot{.} \\
\ddot{.} \\
\ddot{336} \\
(100 \% \text { oxygen) } \\
320 \\
(100 \% \text { oxygen) } \\
\ddot{.} \\
220 \\
(100 \% \text { oxygen) }\end{array}$ & $\begin{array}{l}45-0 \\
62-0 \\
40-0 \\
47 \cdot 0 \\
63-0 \\
37 \cdot 0 \\
22 \cdot 0 \\
29 \cdot 5 \\
57 \cdot 5\end{array}$ & $\begin{array}{l}7.360 \\
7 \cdot 245 \\
7 \cdot 410 \\
7 \cdot 330 \\
7.270 \\
7 \cdot 338 \\
7 \cdot 420 \\
7 \cdot 395 \\
7 \cdot 270 \\
7 \cdot 420 \\
7 \cdot 480 \\
7 \cdot 450\end{array}$ & $\begin{array}{c}0.016 \\
0.016 \\
\ddot{ } \\
\ddot{0} \\
0.017 \\
0 \ddot{0} \\
0.016\end{array}$ \\
\hline
\end{tabular}

Figura 4. Tabla del artículo original en la que se muestran las variables respiratorias evaluadas. Nótese que el ventilador empleados fue el modelo Bennett, como el que se muestra en la figura 2.

\section{TABLE III-EFFECT OF POSITIVE END-EXPIRATORY PRESSURE IN PATIENT 11 WITH VIRAL PNEUMONIA}

\begin{tabular}{|c|c|c|c|}
\hline \multicolumn{2}{|l|}{-} & $\begin{array}{l}\text { No retard } \\
(301 . / \mathrm{min} .)\end{array}$ & $\begin{array}{c}\text { Retard } \\
(15 \mathrm{l} . / \mathrm{min} .)\end{array}$ \\
\hline $\begin{array}{l}\left.\mathrm{P}_{10} \text { (mm. } \mathrm{Hg}\right) \\
\left.\mathrm{PaO}_{2} \text { (mm. } \mathrm{Hg}\right) \\
\mathrm{S}_{\mathrm{aO}_{2}} \text { (\%)... } \\
\mathrm{PaCO}_{2} \text { (mm. } \mathrm{Hg} \text { ) } \\
\mathrm{pH} \quad . . \\
\mathrm{Blood} \text {-pressure (m } \\
\text { Time (min.) }\end{array}$ & 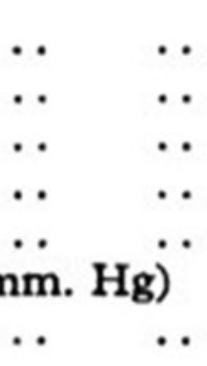 & $\begin{array}{c}560(\mathrm{~mm} . \mathrm{Hg}) \\
42(\mathrm{~mm} . \mathrm{Hg}) \\
78(74) \\
31(33) \\
7.436(7.435) \\
120 / ? \text { (mm. } \mathrm{Hg}) \\
0 \text { (55 min.) }\end{array}$ & $\begin{array}{c}560(\mathrm{~mm} . \mathrm{Hg}) \\
141.5(\mathrm{~mm} . \mathrm{Hg}) \\
98(98) \\
39.5(35) \\
7.370(7.405) \\
120 / 84(\mathrm{~mm} . \mathrm{Hg}) \\
30 \mathrm{~min} .(65 \mathrm{~min} .)\end{array}$ \\
\hline
\end{tabular}

Figura 5. Tabla del artículo original en la que se observan los efectos de la presión positiva al final de la espiración en uno de los enfermos estudiados. Nótese el incremento de la presión parcial de oxígeno en la sangre arterial $\left(\mathrm{PaO}_{2}\right)$ y de la saturación de oxígeno $\left(\mathrm{SaO}_{2}\right)$ a los 30 minutos de iniciada la maniobra.

... "Recovery of respiratory function was complete in 3 patients; all 3 were injury cases, and recovery after continuous positive-pressure respiration was rapid and progressive."

La discusión es magistral. En ella se describen conceptos como inestabilidad alveolar (que a la fecha denominamos atelectrauma y volutrauma), disfunción y alteraciones del surfactante como común denominador de las alteraciones pulmonares en los pacientes descritos, y atelectasias microscópicas (colapso alveolar). Además, se sientan las bases de la PPFE y se enfatiza en que la disminución de la distensibilidad está relacionada con el colapso alveolar, y que por este motivo se requiere una mayor presión para su apertura, sentando las bases iniciales del reclutamiento alveolar y la necesidad de la PPFE. Por último, se señala que el control del disparador de la lesión pulmonar es parte medular del manejo, sin lo cual el resto de las medidas son fútiles:

... "In view of the similar response of the lung to a variety of stimuli, a common mechanism of injury is postulated. The loss of lung compliance, refractory cyanosis, and microscopic atelectasis point to alveolar instability as a like source of trouble. The inability to find obstruction in bronchioles or bronchi would tend to rule out the larger airways as a cause for rapidly decreasing compliance." ... "The inability to measure the surface-active agent directly is a serious obstacle in the effort to link 


\title{
The Adult Respiratory Distress Syndrome* Clinical Features, Factors Influencing Prognosis and Principles of Management
}

\author{
Thomas L. Petty, M.D., F.C.C.P., and David G. Ashbaugh, M.D.
}

Figura 6. Cabecera de la publicación en la que se consolida el concepto del síndrome de insuficiencia respiratoria aguda.

this agent with clinical and pathological states. However, the theoretical basis for its presence is convincing, and indirect measurements seem to indicate that its loss is associated with the development of the clinical, physiological, and pathological conditions seen in the 12 patients in this series."

... "The theoretical basis for positive end-expiratory pressure coincides with the theoretical basis for loss of lung compliance. If surfactant is diminished, alveoli should collapse on expiration when the end-expiratory pressure is at atmospheric levels. Collapsed alveoli require greater pressures for reopening, thus explaining the notable loss of compliance."

..."The use of positive end-expiratory pressure merely buys time: unless the underlying process can be successfully treated or reversed the prognosis is grave."

Posterior a su primera publicación, los Dres. Ashbaugh y Petty continuaron con su línea de trabajo e investigación, que culminó en un artículo publicado en Chest en 1971, titulado The adult respiratory distress syndrome ${ }^{4}$ (Fig. 6).

\section{The adult respiratory distress syndrome}

En este artículo, a partir de la descripción de tres casos clínicos, se consolida el concepto de SIRA como una enfermedad diferente con características definidas y particulares, para la que se hacen recomendaciones de manejo específicas. Las aportaciones de esta publicación son torales en la medicina intensiva y las podemos resumir en lo siguiente:

- La definición y los criterios diagnósticos de una nueva enfermedad.

- La fisiopatología de la enfermedad y el papel del surfactante.

- Los diferentes disparadores, ya sea pulmonares o extrapulmonares.
- El concepto de toxicidad por oxígeno.

- El concepto de lesión pulmonar inducida por ventilación, a la que los autores denominan «pulmón de respirador».

- La descripción de complicaciones y desenlaces.

- La importancia de la implementación de medidas preventivas.

- La importancia del monitoreo del intercambio de gases, la función respiratoria y las curvas de ventilación, y el seguimiento radiológico, durante el manejo de los enfermos (Figs. 7 y 8).

- Los principios de manejo, que se clasificaron en dos grupos: los encaminados a prevenir el colapso alveolar y los dirigidos a evitar una mayor lesión pulmonar. Los autores determinan conceptos fundamentales, entre los que destacan el control en la fracción inspirada de oxígeno, la importancia de la PPFE, la ventilación mecánica con volumen, la restricción de líquidos, el uso adecuado de antibióticos y el probable efecto benéfico de los esteroides, en especial en escenarios especiales (Fig. 9).

De cada uno de los conceptos derivados de sus observaciones, como es bien sabido de todos ustedes, se han escrito a la fecha un gran número de trabajos derivados de una extensa investigación básica, molecular, genética, epidemiológica y clínica, de lo que han derivado el conocimiento actual y los protocolos de diagnóstico y manejo que seguimos en el SIRA. Un buen número de los conceptos de Ashbaugh, Petty y sus colaboradores siguen siendo válidos.

\section{Los autores}

Con mucha frecuencia no conocemos a los autores de los artículos que leemos. Por este motivo nos dedicamos a buscar información de los médicos que 
A insPiratory PLATEAU AND POSITIVE END EXPIRATORY PRESSURE OHIO $\$ 60$

flow
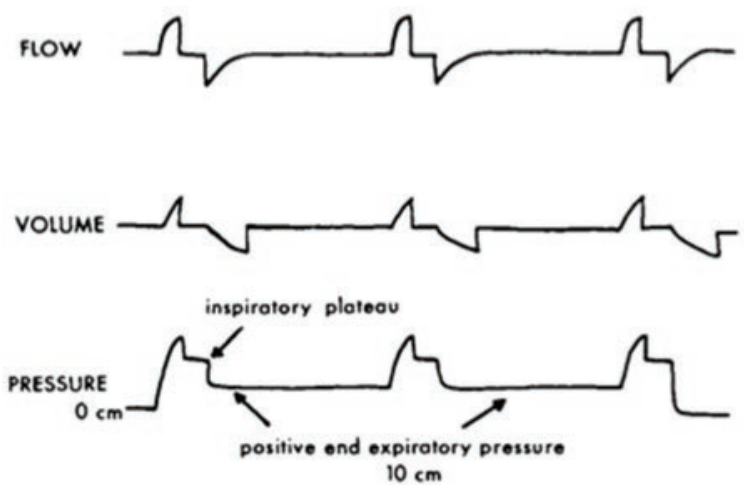

B

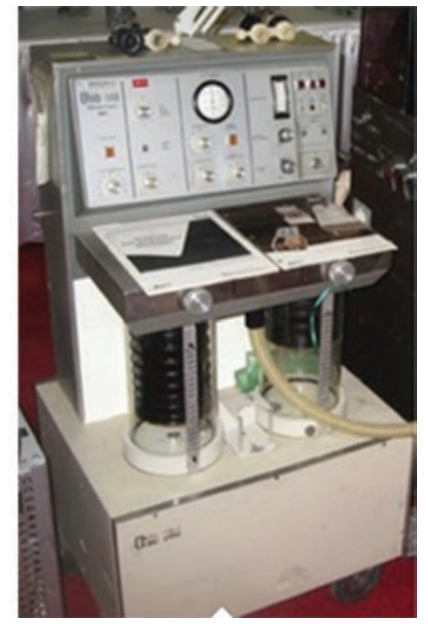

Figura 7. A: curvas de ventilación mecánica en un paciente con síndrome de insuficiencia respiratoria aguda en las que se observa el monitoreo de la presión plateau y de la presión positiva al final de la espiración. B: ventilador Ohio 560 con el que fueron ventilados los pacientes del segundo reporte.

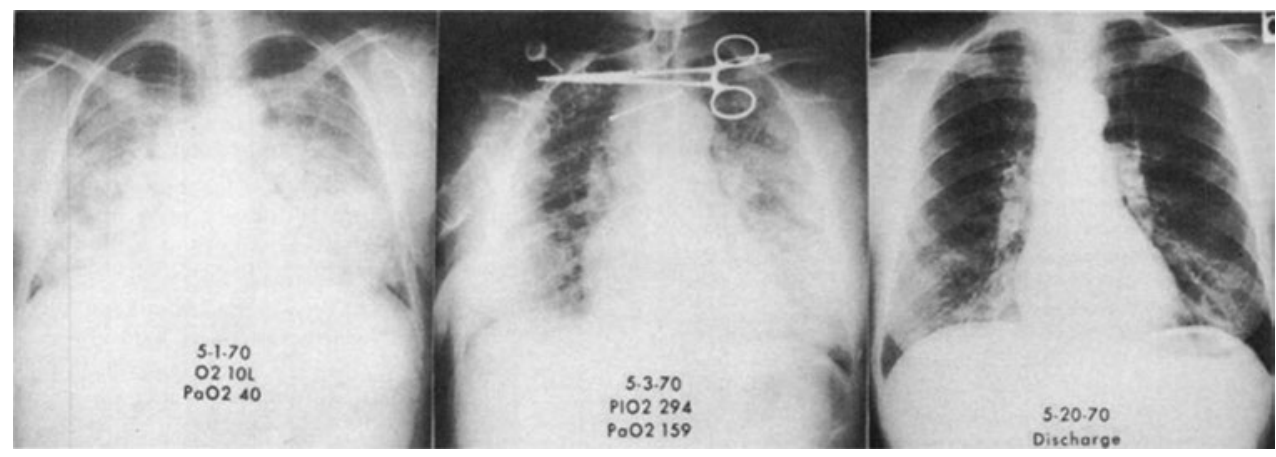

Figura 8. Evolución radiográfica de los infiltrados de un paciente con síndrome de insuficiencia respiratoria aguda. Nótese su involución posterior a la implementación de la ventilación mecánica y la presión positiva al final de la espiración hasta el alta del enfermo.

publicaron el artículo que nos ocupa, aunque cabe señalar que no hay mucha información de ellos en los medios electrónicos. De los Dres. Levine y Bigelow no encontramos muchos datos. Sabemos que eran neumólogos, que formaban parte de la Sociedad de Tuberculosis y Enfermedades del Tórax de los EE.UU., y que eran parte del equipo del Centro Médico de la Universidad de Colorado. Ambos fueron residentes del Dr. Thomas Petty en el Hospital General de la Universidad de Colorado, y junto con él echaron a andar en 1965 el servicio de cuidados respiratorios (neumología y terapia respiratoria). Es un hecho importante relatar que el primer enfermo que atendieron en ese servicio fue un paciente de edad avanzada, de nombre Earl, que ingresó con un cuadro de enfermedad pulmonar obstructiva crónica (EPOC) por enfisema descompensado. El equipo puso manos a la obra y se organizaron de la siguiente manera: el Dr. Ashbaugh, jefe de residentes de cirugía, realizó la traqueotomía; los Dres. Bigellow y Levine conectaron al enfermo a un ventilador Bird Mark VII (el que nosotros llamamos cariñosamente «la cajita verde»); y el Dr. Petty tomo la gasometría y procesó la determinación de los gases con un dispositivo dotado de los electrodos de Clark y Severinghaus. Como comentó el mismo Dr. Petty: "la noche fue larga, el paciente se recuperó, pero la historia ya no fue la misma». En esos momentos se integró el grupo que 2 años después publicaría el artículo que revolucionó la medicina intensiva, los cuidados respiratorios y la ventilación mecánica. Los Dres. Petty y Ashbaugh, internista neumólogo y cirujano, trabajaron estrechamente en los siguientes años, mientras que el Dr. Levine se especializó en la medición de la tensión de superficie del sobrenadante del líquido alveolar y del surfactante. El Dr. Levine radicaba en Phoenix, Arizona, al momento de la publicación.

El Dr. Ashbaugh, primer autor del artículo, nació en el Estado de Ohio y estudió medicina en la Universidad de Colorado. Se especializó en cirugía cardiotorácica. Conoció al Dr. Thomas Petty durante la residencia. Además de la cirugía, tuvo gran interés en el estudio 


\section{Table 4-Management Principles in ARDS}

\section{Prevent alveolar collapese and inaintain oxyenation \\ a Tracheostomy \\ b Volume respirator \\ i. Oxygen control \\ d Pesitive end-expiratory prexiure (l'F,F, )}

\section{Prevent further injury \\ a ()xygen control \\ b Fluid restriction \\ - Antibiotics for sperific infections \\ d Corticosteroid drugs}

Figura 9. Tabla tomada del artículo original en la que se muestran los principios de manejo del síndrome de insuficiencia respiratoria aguda de acuerdo con Petty y Ashbaugh.

de los enfermos con lesión pulmonar aguda desarrollada tras una lesión primaria y secundaria, un tipo especial de edema agudo pulmonar al que denominó «no cardiogénico». Trabajó como cirujano, profesor e investigador en el Centro Médico de la Escuela de Medicina de la Universidad de Washington. Hizo práctica privada en Boise, Idaho, durante 15 años. Al jubilarse de su actividad profesional se retiró a la Isla López.

Del grupo de trabajo que colaboró en la publicación, de quien más se ha escrito y se conoce en los anales históricos es del Dr. Thomas L. Petty, debido a sus múltiples contribuciones a la medicina intensiva y la neumología. El Dr. Petty nació en Boulder, Colorado, el 23 de diciembre de 1932, y murió a los 76 años de edad el 12 de diciembre de 2009. Estudió en la Universidad de Colorado, donde obtuvo el grado de doctor en medicina en 1958 graduándose con honores. Su internado lo realizó en el Hospital General de Filadelfia, y la residencia la hizo en Michigan y en Colorado. Fue jefe de residentes de medicina interna en el Hospital General de la Universidad de Colorado. Fue ahí donde conoció al Dr. Ashbaugh, quien era jefe de residentes de cirugía.

El Dr. Petty organizó y dio inició a los cuidados respiratorios intensivos y multidisciplinarios, implementó el monitoreo en las unidades de terapia intensiva, en especial hizo énfasis en la importancia de la determinación de los gases arteriales, impulsó el desarrollo de la ventilación mecánica, introdujo la rehabilitación respiratoria en pacientes con enfermedades pulmonares, en especial con EPOC, e implementó los cuidados respiratorios y la oxigenoterapia domiciliarios. Se le consideró el mejor neumólogo de su generación (fue considerado el padre de la neumología moderna) y quien más aportaciones dio a este campo de la medicina. Investigador dedicado y escritor prolijo, publicó y editó 45 libros y 800 artículos originales, además de numerosos folletos educativos dirigidos tanto a médicos como a pacientes y sus familiares. Cuentan los que le conocieron que llegaba a su oficina a las 5 de la mañana y escribía de 2 a 3 horas, para continuar luego su trabajo con la visita clínica, la enseñanza, los protocolos de investigación y la dedicación a sus enfermos. Por si fuera poco, fue editor de las principales revistas de neumología, medicina interna y medicina intensiva de su época. Desarrolló programas educativos dirigidos a los enfermos, en especial a los de EPOC.

Hubo dos hechos importantes en la vida del Dr. Petty: 1) siendo residente fue castigado y suspendido varios días por haber tomado una muestra de sangre arterial a un paciente para el análisis de los gases arteriales; y 2) la pregunta de siempre, ¿por qué, si la cabeza del grupo era él, el primer autor del artículo en 

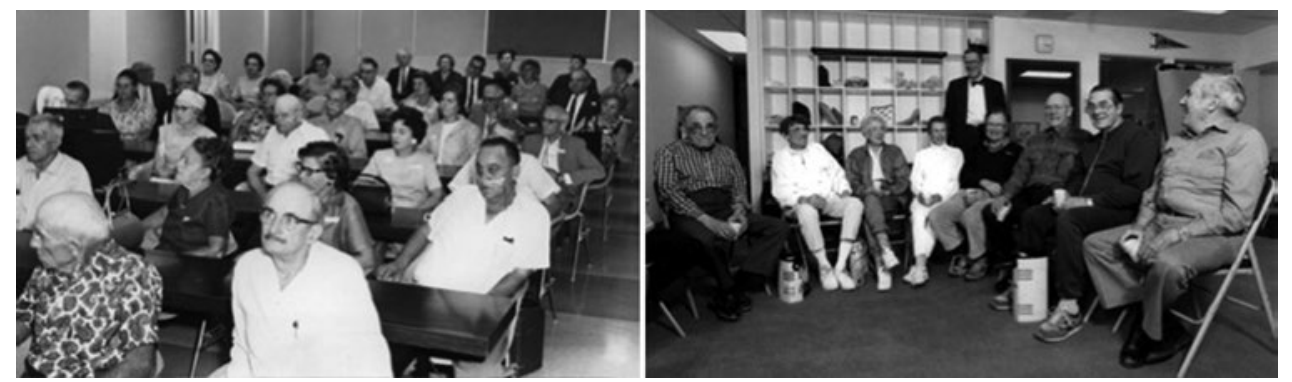

Figura 10. Clínicas de enfermedad pulmonar obstructiva crónica creadas y dirigidas por el Dr. Petty. Pueden verse algunos enfermos con un dispositivo de aporte de oxígeno administrado por puntas nasales. En la fotografía de abajo, el Dr. Petty al fondo, de traje y con corbata de moño.

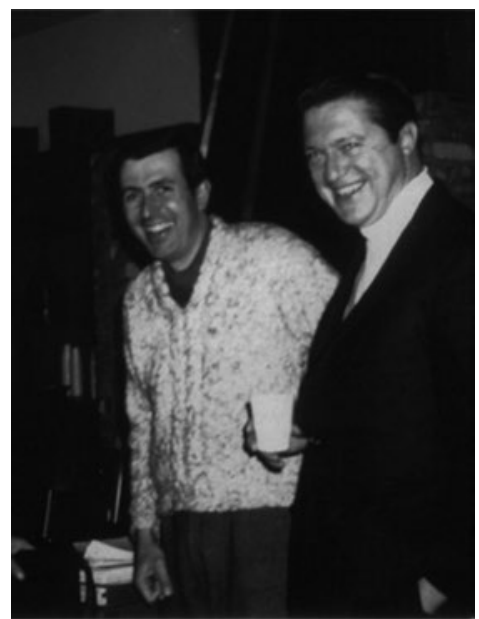

Figura 11. Foto histórica de finales de la década de 1960. A la derecha, con un vaso en la mano, el Dr. Thomas L. Petty, y a la izquierda, con suéter de color claro, el cirujano cardiotorácico David G. Ashbaugh.

que se describe el SIRA es el Dr. Ashbaugh? Esto fue una decisión del mismo Dr. Petty, quien reconoció que el primer paciente que manejaron era un caso del Dr. Ashbaugh, y por la estrecha colaboración y entusiasmo de este último en la integración de la muestra, el conseguir los ventiladores, el análisis de los resultados y la publicación misma. Este hecho refleja la bonhomía del Dr. Petty. A pesar de lo anterior, en todos los círculos científicos se reconoce al Dr. Petty como el de la idea genial de describir una nueva enfermedad.

Su principal diversión era organizar excursiones para ir de pesca. Decía, cuando preparaba con sus amigos y residentes estos paseos, que la diversión estaba dividida de la siguiente manera: « $25 \%$ al planearla, $50 \%$ al estar pescando, $25 \%$ al recordarla y ver las fotografías». En pocas palabras, el Dr. Petty fue un gran hombre, humanista, médico, investigador, maestro y pescador (Figs. 10 y 11).

\section{Conclusiones}

El estudio del SIRA es fascinante; desde su descripción inicial y en los siguientes 50 años, los estudios en modelos animales, los ensayos clínicos controlados, la genética, la biología molecular, la imagenología, la bioingeniería y los avances en ventilación mecánica nos han abierto un amplio panorama, siendo el inicio del camino la magistral y original publicación del 12 de agosto de 1967 por los Dres. Ashbaugh, Petty, Bigellow y Levine. Seguramente nunca imaginaron la trascendencia de su publicación, la gran cantidad de investigación y publicaciones que se derivaron de ella, la rápida evolución de la ventilación mecánica posterior a la introducción de la PPFE y de los conceptos de ventilación por volumen y presión, el conocimiento de la lesión pulmonar asociada a la ventilación, el número de vidas que se han salvado y, en especial, que fueron los forjadores de una nueva época en la medicina intensiva.

Sea este escrito un homenaje a tan geniales médicos, pero en especial al Dr. Thomas L. Petty.

\section{Conflicto de intereses}

Los autores declaran que no tienen conflicto de intereses bajo ningún rubro.

\section{Bibliografía}

1. Ashbaugh DG, Bigelow BD, Petty TL, Levine BE. Acute respiratory distress in adults. Lancet. 1967;2:319-23.

2. Slutsky AS. History of mechanical ventilation. From Vesalius to ventilation-induced lung injury. Am J Respir Crit Care Med. 2015;191:1106-15.

3. Ranieri VM, Rubenfiled GD, Thompson BT, Ferguson ND, Caldwell E, Fan E, et al. Acute respiratory distress syndrome: the Berlin definition. JAMA. 2012;307:2526-33.

4. Petty TL, Ashbaugh DG. The adult respiratory distress syndrome. Chest. 1971;60:233-9. 